\title{
The need to clarify the multiple roles played by nurses in the face of challenges posed by terrorist attacks
}

\author{
Kanae TANAKA, Yumiko OGAWA and Yoko SUZUKI \\ Doctoral Students of Disaster Nursing Global Leader Degree Program, Cooperative Doctoral Course in Disaster Nursing, Graduate School \\ of Health Care Sciences, Tokyo Medical and Dental University, Tokyo, Japan
}

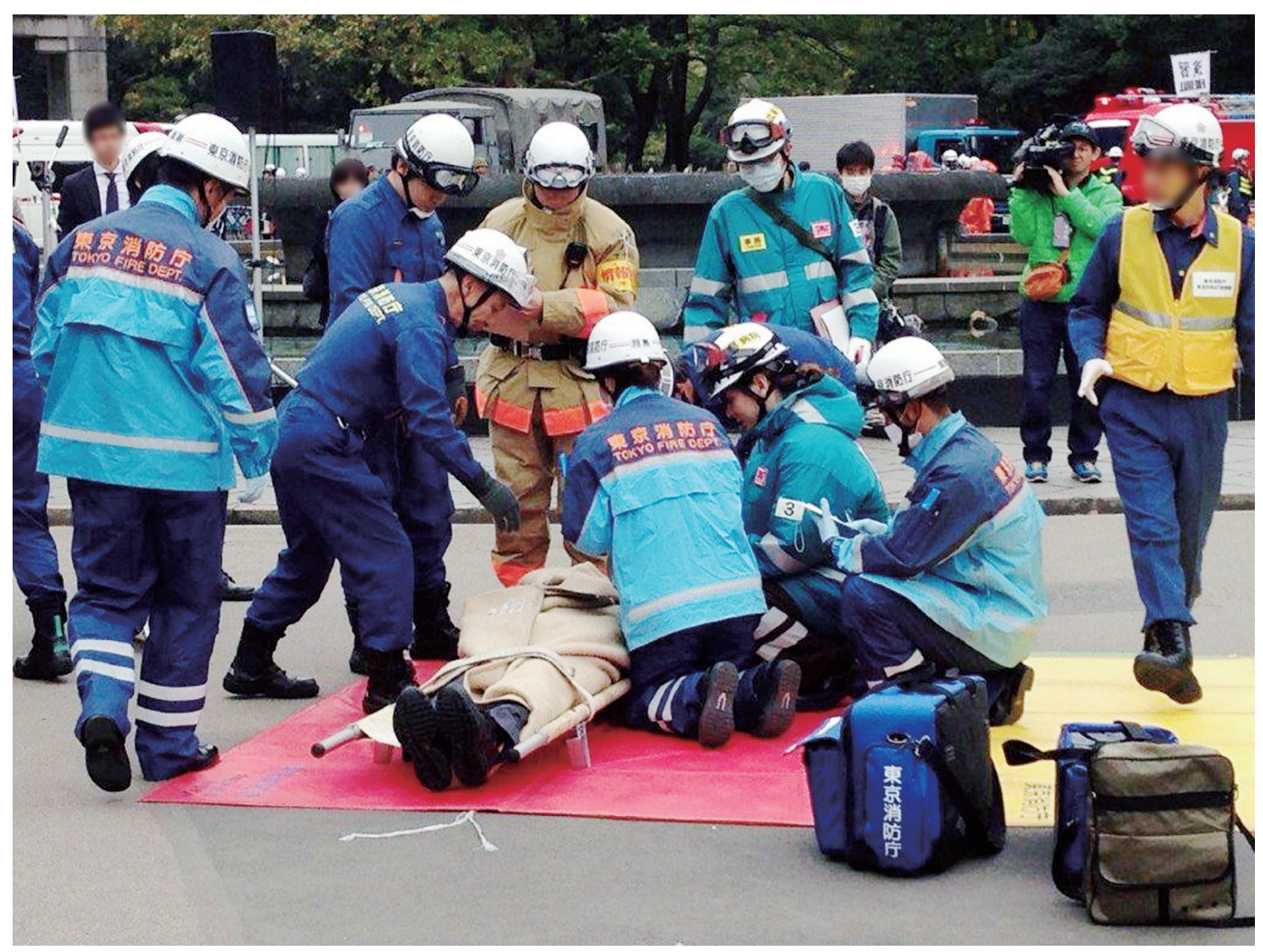

Late in the fall of 2014, under a wintery sky, a large-scale terrorism training session was carried out at a public park in central Tokyo. During the drill, firefighters, paramedics, police, the Self Defense Forces, Tokyo Disaster Medical Assistance Team (DMAT), subway station employees, Tokyo government officials, and staff of

Correspondence: Kanae Tanaka, Tokyo Medical and Dental University, 2-3-10 Kandasurugadai Chiyoda-ku Tokyo 101-0062, Japan. Email: dn140003@tmd.ac.jp

Received 21 January 2015; accepted 27 February 2015. private enterprises around the park practised coping with a chemical attack that simulated the March 20, 1995 sarin gas attack on a Tokyo subway.

The scenario started with passengers encountering a suspicious substance in an underground subway passage. The authors, who are doctoral students majoring in disaster nursing, and private enterprise staff participated in the drill as evacuees, and subway station employees guided them to safety. Rescue and response teams comprised various organizations who worked together to triage the casualties, decontaminate victims, help them 
to see DMAT, and transfer the injured to hospitals, etc. This marked the first trial focusing on the collaboration between the two primary subway companies in Tokyo and other rescue and response teams. The teams seemed to work well together and we learned a lot from this drill; we were especially impressed by the rapid response time of the emergency teams and how reliable and helpful the equipment was and the sophisticated command system used by the multi-disciplinary team. However, we suggest that more realistic training, such as a greater number of casualties, greater collaboration with people living or working around the site or blind drills be conducted in the future. First, we expect another drill that involves more casualties (e.g., an explosive attack on the Tokyo Marathon, an event attracting 36,000 runners and more than 1,300,000 spectators), which will be coordinated by rescue and response teams.

In addition, during the present scenario, there were relatively few nurses involved compared to the numbers that would be deployed in real scenarios. In this training, only Tokyo DMAT issued nurses, and they provided nursing care only at the first-aid station. We believe nurses can greatly contribute to various areas including decontamination and triage. Nurses can secure the safety and privacy of casualties, and give words of encouragement to victims and concerned family members. We can provide victims with appropriate care, even during terrorist attacks. More active transmission of information about the role of nurses and their ability to cooperate with other professionals is needed.

Finally, Tokyo is a major city and the threat of a terrorist attack is ever present, as is the case with other metropolitan cities around the world. We should prepare for not only chemical attacks, but also biological, radiological, and nuclear attacks, as well as explosive events. To minimize casualties in such events, the roles played by nurses in responding to various aspects of disaster and in cooperating with other experts should be made clear in order to prepare for all types of attacks.

We did not have any financial support or relationships that may pose a conflict of interest.

Key words: nurse's role, terrorism, training 